\title{
Simulation Models of the Electromagnetic Forming Process
}

\author{
G. Bartels, W. Schaetzing, H.-P. Scheibe And M. Leone \\ Institute of Fundamental Electrical Engineering and Electromagnetic Compatibility \\ University of Magdeburg, Germany
}

\begin{abstract}
Suitable simulation approaches for the preliminary investigation of the electromagnetic metal forming process are presented. For this purpose an uncoupled (loose-coupled) simulation model is compared to a more rigorous sequential-coupled approach. As shown by examples, the connection between electromagnetic field and spatiotemporal evolution of the deformation of the workpiece cannot always be neglected to describe the electromagnetic forming process with adequate accuracy.
\end{abstract}

PACS numbers: 02.60.-x, 02.60.Cb, 02.70.-c, 02.70.Dh, 13.40.-f, 52.40.Db

\section{Introduction}

The electromagnetic forming (EMF) process is a high-dynamic process with high transformation velocities and was developed in the 1960s. A typical application area is the electromagnetic tube compression. As shown in Fig. 1, the arrangement consists of an electromagnetic forming coil and an electrically conductive tubular workpiece. The deformation of the tube results from discharging a capacitor $\mathrm{C}$ by closing the switch $\mathrm{S}$, leading to the interaction (the Lorentz force) between the electrical current in the forming coil (copper) and the induced eddy current in the tubular workpiece [1].

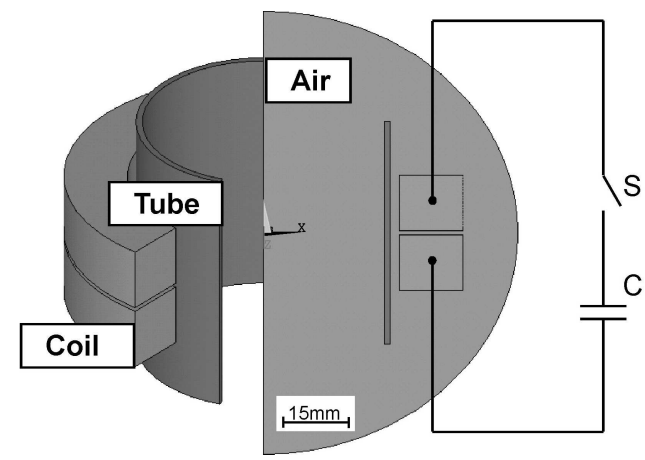

Fig. 1. Arrangement for electromagnetic tube compression.

\section{Simulation models}

In the electrical engineering complicated electromagnetic field problems are increasingly solved by numerical computation methods. In this work the finite element method (FEM) software ANSYS was used. Figure 2 shows the flowchart of the loose- (a) and of the sequential-coupled algorithm (b), respectively, as applied for the simulation of the electromagnetic metal forming process. In the loose-coupled approach the electromagnetic field problem is solved in the time domain first by solving Eq. (1) based on Maxwell's equations, where $\boldsymbol{A}$ is the magnetic vector potential, $\boldsymbol{J}$ the current density, $[L]$ and $[K]$ are the conductivity and permeability matrix, respectively, and $\varphi$ the electric scalar potential

$$
[L(\gamma)]\left[\begin{array}{c}
\frac{\partial}{\partial t} \boldsymbol{A} \\
\varphi
\end{array}\right]+[K(\mu)]\left[\begin{array}{c}
\boldsymbol{A} \\
0
\end{array}\right]=\left[\begin{array}{l}
\boldsymbol{J} \\
0
\end{array}\right] .
$$

The computation of the structure-mechanical transforming process is calculated in a subsequent mechanical simulation by solving the fundamental motion equation

$$
[M]\left\{u^{\prime \prime}\right\}+[D]\left\{u^{\prime}\right\}+[C]\{u\}=\{F(t)\} .
$$

Equation (2) includes the mass matrix $[M]$, the damping matrix $[D]$ and the stiffness matrix $[C]$. The node displacements are represented by the vector $\{u\}$, while $\left\{u^{\prime}\right\}$ denotes the node-velocity and $\left\{u^{\prime \prime}\right\}$ the node-acceleration vector, respectively. $\{F(t)\}$ represents the load vector. In the sequential-coupled simulation model the electromagnetic field simulation and the structure-mechanical simulation are iteratively performed, taking into account the time dependent geometry of the workpiece. The FEM model used for the analysis of the 2D axial symmetrical geometry is shown in Fig. 3. For the mesh 8-node elements are used "plane53" in the electromagnetic model based on the magnetic vector potential formulation and is applicable for magnetic field analyses. In the mechanical simulation the same mesh is used with the properties of "plane82". The discharge circuit is modeled by an equivalent circuit (Fig. 3a), which includes the interconnect inductance $L=32.6 \mathrm{nH}$ and ohmic resistance $R=1.8 \mathrm{~m} \Omega$ between the capacitor $\mathrm{C}$ and the coil. M1 and M2 are elements to couple the circuit with the turns of the coil in the FEM model. Two different discharge configuration with equal energy $\left(W_{\mathrm{el}}=1.2 \mathrm{~kJ}\right)$ were con- 
sidered. In system 1 the capacitance and charging voltage $(U)$ are $C_{1}=240 \mu \mathrm{F}$ and $U_{1}=3162 \mathrm{~V}$. System 2 has $C_{2}=1440 \mu \mathrm{F}, U_{2}=1291 \mathrm{~V}$. A simulation time of $72 \mu \mathrm{s}$ was chosen, with a time step of $1 \mu \mathrm{s}$. The material behaviour of the tube (AA6063-T4, $\varnothing=60 \mathrm{~mm}$, thickness $=1.5 \mathrm{~mm}$, length $=60 \mathrm{~mm})$ is considered by the true stress-strain curve.



Fig. 2. (a) Flowchart of the loose-coupled algorithm, (b) flowchart of the sequential-coupled algorithm.

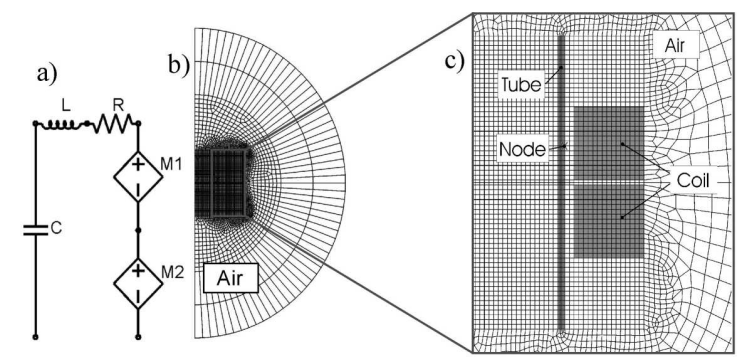

Fig. 3. (a) Equivalent circuit; (b) FEM model, (c) detailed view of the FEM model with selected node.

\section{Results and discussion}

Figure 4 shows the results of the loose-coupled and the sequential-coupled simulation approaches, for the two chosen system configurations. In Fig. 4a the time-dependent current through the coil is plotted for system 1 and 2. As expected, system 1 has about a twice larger discharge frequency, due to the larger value of the capacitor. For a selected node on the tube surface (Fig. 3c), the resulting time-dependent magnetic pressure and the radial displacement are shown in Fig. 4b and Fig. 4c, respectively. As can be seen, the deviations between the two simulation approaches increase with time, especially for the slower system 2 . The loose-coupled approach leads to an overestimation of the final deformation, as shown in Fig. 4d [2]. The reason is that the coil current and in turn also the magnetic pressure remain unaffected from the changing tube geometry, while in the sequential-coupled algorithm it is taken into account. The simulation results clearly show that the slower discharge system 2 provides larger final deformations [3] in comparison with the faster system 1 , for equal load energy.

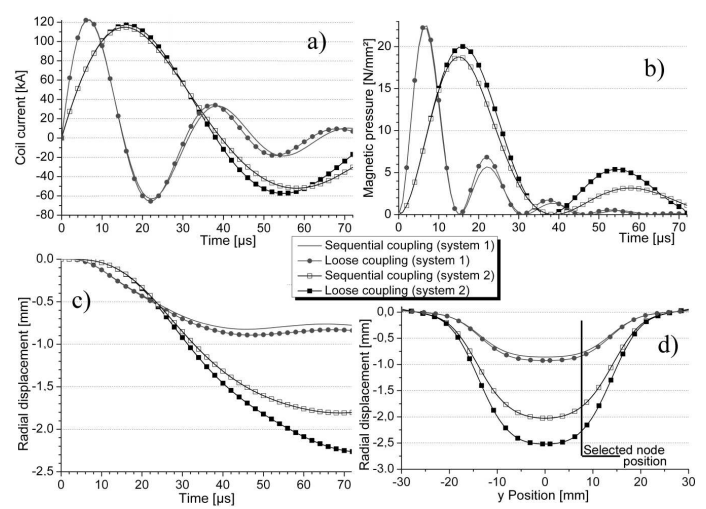

Fig. 4. (a) Coil current vs. time, (b) magnetic pressure vs. time (selected node), (c) radial displacement vs. time (selected node), (d) radial displacement of the tube surface at simulation end.

\section{Conclusions}

For the simulation of the electromagnetic metal forming process two different approaches were presented, one with loose-coupled and the other with a sequentialcoupled between the electromagnetic field and mechanical deformation. As shown by a representative example with a tubular workpiece and two different discharge velocities, the deviations between the two approaches increase with time and may yield considerable overestimations of the loose-coupled model. Therefore, the simple loose-coupled approach can only be used for relatively fast deformation processes. Otherwise the more accurate sequential-coupled model should be used.

\section{References}

[1] R. Winkler, Hochgeschwindigkeitsbearbeitung, Berlin 1973.

[2] Y.U. Haiping, L.I. Chufeng, D.E.N.G. Jianghua, Haiping, J. Mat. Process. Tech. 209, 707 (2009).

[3] Y.U. Haiping, L.I. Chufeng, J. Mater. Process. Tech. 209, 1053 (2009). 